\title{
ENTROPY OF MIXING OF NaCd AND AIMg MOLTEN ALLOYS
}

\author{
S.K. Chatterjee*,* L.C. Prasad* and A. Bhattarai** \\ **Department of Chemistry, Mahendra Morang Adrash Multiple Campus, Biratnagar, Tribhuvan University, Nepal. \\ *P G Department of Chemistry, T M Bhagalpur University, Bhagalpur 812 007, India.
}

\begin{abstract}
The complex formation model is used to explain the anomalous behaviour of entropy of mixing of $\mathrm{NaCd}$ and $\mathrm{AlMg}$ liquid alloys as a function of concentration. The interionic pair potential $\phi_{i j}(r)$ evaluated within the framework of pseudopotential theory which in turn is used to obtain the values of hard-sphere diameter of $\mathrm{NaCd}$ and $\mathrm{AlMg}$ liquid alloys. The hard-sphere diameter is used to evaluate the concentration dependent anamoly in entropy of mixing which occurs due to preferential ordering of unlike atoms as nearest neighbour on $\mathrm{NaCd}$ and $\mathrm{AlMg}$ liquid alloys, which could be simultaneously understood with the help of complex formation model. The computed value of Entropy of mixing $\left(\mathrm{S}_{\mathrm{M}}\right)$ from pseudopotential theory is positive at all concentration range except $0.8 \leq C_{\mathrm{cd}} \leq 0.9 \mathrm{in} \mathrm{NaCd}$ liquid alloys. The disagreement between theory and experiment might be due to parameterisation of hard- sphere diameter of the complex $\left(\sigma_{3}\right)$ and $\psi_{\text {comp }}$.
\end{abstract}

Key Words: Entropy of mixing; Pseudopotential theory; Hard-sphere diameter; Pair-Potential.

\section{INTRODUCTION}

Various theoretical models ${ }^{1-6}$, based on the formation intermetallic compounds, pseudomolecules, complexes in the melt are used to study the energetics of strong interacting binary liquid alloys. Stronger interaction ${ }^{7}$ corresponds to greater possibility of compound formation. The first principal theory in the framework of pseudopotential formalism can be used to calculate the pair-potential which subsequently, can be used to study the interionic interaction ${ }^{8-10}$, but pseudopotential theory, itself suffers from limitations. For those alloys, whose mixing properties exhibit anomalous behaviour due to strong interaction between the unlike atoms leading to compound formation, pseudopotential theory cannot be applied with confidence $e^{7,8,11}$. In view of limitation, the pseudopotential theory requires improvement, so that it could be applied strongly to such alloys.

In this work an attempt has been made for study the entropy of mixing of $\mathrm{NaCd}$ and $\mathrm{AlMg}$ alloys as a function of concentration on the basis of pseudopotential formalism.

Hoshino and Young ${ }^{11-13}$ have used the hard-sphere model to compute the entropy of mixing. The hard -sphere diameter $(\sigma)$ evaluated from interionic pair potential $\phi_{i j}(r)$ which is evaluated in particular from the band structure energy of pseudopotential theory ${ }^{7-10}$. The concentration dependent thermodynamic properties ${ }^{14-17}$ of liquid $\mathrm{NaCd}$ and AlMg alloys have been reported interesting features. Thermodynamical ${ }^{16-17,25}$ properties were deviated maximally from the ideal values around the concentration close to the configuration $\mathrm{Cd}_{2} \mathrm{Na}$ and $\mathrm{Al}_{3} \mathrm{Mg}_{2}$.

\section{COMPLEX FORMATION MODEL FOR ENTROPY OF MIXING}

\subsection{FORMALISM}

The properties of many binary liquid alloys exhibit a pronounced asymmetry as a function of concentration even though the size effect is not large enough to cause the asymmetry. These liquid alloys have the characteristics that in the solid state that they form complexes, at some stoichiometric composition. Therefore, it is tempting to assume that such chemical complexes or pseudomolecules $\left(\mu A+v B=A_{\mu} B_{v}, \mathrm{~A}\right.$ and $\mathrm{B}$ are constituent atoms, $\mu$ and $v$ are very small integers) also exists in the liquid phase, close to the melting temperature. The model explains successfully the concentration fluctuation in the long wavelength limit and thermodynamic properties of a number of binary liquid alloys $2,4,6,18-22,25$

This encourages us to study the entropy of mixing of AlMg and $\mathrm{NaCd}$ liquid alloys as a function of concentration.

Author for Correspondence: S.K. Chatterjee, Department of Chemistry, Mahendra Morang Adrash Multiple Campus, Biratnagar, Tribhuvan University, Nepal.E-mail: sujeetkumarchatterjee@yahoo.com. 
A binary liquid alloys containing in all $N_{\mathrm{A}}[=N(1-\mathrm{C})]$ of atoms A and $N_{\mathrm{B}}[=(N C)]$ of atoms of B may be assumed to consists of $n_{\mathrm{m}}\left(=\mathrm{n}_{3} N\right)$ complexes, which also acts as independent scattering centres to the alloy, the total number of scattering points are

$N_{\mathrm{s}}=n_{\mathrm{A}}+n_{\mathrm{B}}+n_{\mathrm{m}}=n N$

Where $n=n_{1}+n_{2}+n_{3}$

From the conservation of atoms we have

$n_{\mathrm{A}}+\mu n_{\mathrm{m}}=N_{\mathrm{A}}$

$n_{\mathrm{B}}+n_{\mathrm{m}}=N_{\mathrm{B}}$

$n_{1}=1-\mathrm{C}-n_{3}$

$n_{2}=C-n_{3}$

$n=1-(+-1) n_{3}$

Here $C$ is the concentration of the second species.

The volume in which $n_{\mathrm{A}}$ atoms of $A$ and $n_{\mathrm{B}}$ atoms of $B$ are randomly distributed and can be expressed as;

$\Omega=\Omega_{\text {alloy }}-n_{m} V_{m}$

Where $\Omega_{\text {alloy }}$ is the atomic volume of the alloy and $V_{\mathrm{m}}$ is the volume of the complex.

$V_{m}=\mu \Omega_{1}+v \Omega_{2}$

Where $\Omega_{1}$ and $\Omega_{2}$ are the atomic volumes of first and second species of the alloys respectively.

The value of $\sigma_{i}$ (hard sphere diameter) as a function of concentration have been determined by minimizing the interionic pair potential $\phi$, i.e.

$\phi\left(\sigma_{1}\right)=\frac{3}{2} K_{B} T+\varphi_{\min }$

where $\frac{3}{2} K_{B} T$ is the mean kinetic energy and $\varphi_{\min }$ is the depth of first minimum in the interionic pair potential.

Using the complex formation model we can express the entropy of mixing forming liquid alloys as ${ }^{11,23}$,

$S_{\mathrm{M}}=S_{\text {gas }}+S_{\mathrm{C}}+S_{\mathrm{n}}+S_{\sigma}+S_{\text {comp }}$

The term $S_{\text {gas }}$ is given by:

$\frac{S_{\mathrm{gas}}}{K_{\mathrm{B}}}=n\left[2.5+\ln \left\{\Omega \mathrm{s}_{\mathrm{c}}\left(\frac{m_{1}{ }^{x 1} m_{2}{ }^{x_{2}} m_{3}{ }^{x_{3}} K_{\mathrm{B}} T}{2 \Pi h^{2}}\right)^{\frac{3}{2}}\right\}\right]$

Here $m_{1}, m_{2}, m_{3}$ are masses of the first atom, second atom and the complex, $K_{\mathrm{B}}$ is the Boltzman constant and $T$ is the absolute temperature. $S_{\mathrm{c}}$ is the ideal mixing term.
$\frac{S_{\mathrm{c}}}{K_{\mathrm{B}}}=-n\left(x_{1} \ln x_{1}+x_{2} \ln x_{2}+x_{3} \ln x_{3}\right)$

Where $x_{i}(i=1,2,3)$ are the concentration fraction of difference scattering centres. The total packing term $S_{\eta}$ is given by

$\frac{S_{\eta}}{K_{\mathrm{B}}}=-n(r-1)(r+3)$

With $r=\frac{1}{1-\eta}$

$\eta$ is the packing density defined as

$\eta=\frac{\Pi}{6 \Omega_{\text {alloy }}}\left(x_{1} \sigma_{1}{ }^{3}+x_{2} \sigma_{2}{ }^{3}+x_{3} \sigma_{3}{ }^{3}\right)$

Where $\sigma_{1}, \sigma_{2}$ are diameter of the species 1 and 2 and $\sigma_{3}$ is the diameter of the complex.

$S_{\sigma}$ arise, due to the difference among the diameters of the free atoms and the complex and is given by

$\frac{S_{\sigma}}{K_{\mathrm{B}}}=S_{\sigma}(1,2)+S_{\sigma}(1,3)+S_{\sigma}(2,3)$

Where

$S \sigma(i, j)=X_{\mathrm{i}} X_{\mathrm{j}}\left(\sigma_{\mathrm{i}}-\sigma_{3}\right)^{2}$

$\left\{[r(r-1)-\ln r]\left(\beta_{1}{ }^{\mathrm{ij}}+\beta_{2}{ }^{\mathrm{ij}}\right)+3(r-1) \beta_{1}^{\mathrm{ij}}\right\}$

$\beta_{1}{ }^{\mathrm{ij}}=\frac{\sigma_{\mathrm{i}}+\sigma_{\mathrm{j}}}{\sigma^{3}}$

$\beta_{2}{ }^{\mathrm{ij}}=\sigma_{\mathrm{i}} \cdot \sigma_{\mathrm{j}}\left(x_{1} \sigma_{1}{ }^{2}+x_{2} \sigma_{2}{ }^{2}+x_{3} \sigma_{3}{ }^{2}\right) / \sigma^{6}$

$\sigma^{3}=\left(x_{1} \sigma_{1}^{3}+x_{2} \sigma_{2}^{3}+x_{3} \sigma_{3}^{3}\right)$

$S_{\text {comp }}$ is the contribution from the internal degrees of freedom of the complexes which is due to the internal modes of vibration. It is approximated by a sum of contribution from each complex, $\psi_{\text {comp }}$ and this may be written as

$\frac{S_{\text {comp }}}{K_{\mathrm{B}}}=n_{3} \Psi_{\text {comp }}$

In view of expression (6), the entropy of mixing of complex forming liquid alloys can be expressed as

$\Delta S_{\mathrm{M}}=\Delta S_{\mathrm{gas}}+\Delta S_{\eta}+S_{\mathrm{c}}+S_{\sigma}+S_{\mathrm{comp}}$

Where

$\Delta S_{\mathrm{i}}=\Delta S_{\mathrm{i}, \text { alloys }}-(1-C) S_{\mathrm{i} 1}-C S_{\mathrm{i} 2}(\mathrm{i}=$ gas,$\eta)$

Where $S_{1}$ and $S_{2}$ are entropies of pure compounds, whose explicit expression can be obtained from above by setting either $C=0$ or $C=1$. 


\section{RESULTS AND DISCUSSION}

The entropy of mixing of compound forming alloys $\mathrm{NaCd}$ and AlMg is computed using equation (14). The packing density $(\eta)$ used for this computation has been obtained by using equation (10). The hard-sphere diameters $\sigma_{1}, \sigma_{2}(1,2=$ constituent atoms used for the computation are taken from Tables 1 and 2). The value of $\sigma_{3}$ (hard sphere diameter for the complex) is fixed by adjusting its value through equation (12) so that a good fit for $S_{\mathrm{M}}$ (experimental) value is obtained. The respective values of $\sigma_{3}$ thus evaluated as 6.56 and 6.51 respectively, for $\mathrm{NaCd}(\mathrm{T}=673 \mathrm{~K})$ and $\mathrm{AlMg}(\mathrm{T}=1073 \mathrm{~K})$ systems.

$\psi_{\text {comp }}$ appearing in equation (13) can be evaluated from the work of Hertz-berg ${ }^{24}$.

Table 1: Volume,diameter and hard-sphere diameter for $\mathrm{NaCd}$ alloys at $673 \mathrm{~K}$.

\begin{tabular}{|l|l|l|l|}
\hline \multicolumn{1}{|c|}{$\boldsymbol{C}_{\mathrm{Cd}}$} & \multicolumn{1}{|c|}{ Volume } & \multicolumn{1}{c|}{$\boldsymbol{\sigma}_{\mathrm{Cd}}$} & \multicolumn{1}{c|}{$\boldsymbol{\sigma}_{\mathrm{Na}}$} \\
\hline 0.0 & 300.616 & - & 6.130 \\
\hline 0.1 & 274.817 & 5.085 & 6.145 \\
\hline 0.2 & 252.384 & 5.013 & 6.157 \\
\hline 0.3 & 232.192 & 5.124 & 6.164 \\
\hline 0.4 & 214.245 & 5.147 & 6.167 \\
\hline 0.5 & 197.420 & 5.168 & 6.166 \\
\hline 0.6 & 182.838 & 5.186 & 6.162 \\
\hline 0.7 & 170.799 & 5.200 & 6.155 \\
\hline 0.8 & 162.650 & 5.210 & 6.149 \\
\hline 0.9 & 158.160 & 5.216 & 6.143 \\
\hline 1.0 & 158.160 & 5.219 & - \\
\hline
\end{tabular}

*Hoshino et. al. (1982), volume at $673 \mathrm{~K}$.

Table 2: Volume,diameter and hard-sphere diameter for AlMg alloys at $1000 \mathrm{~K}$.

\begin{tabular}{|l|l|l|l|}
\hline \multicolumn{1}{|c|}{$\boldsymbol{C}_{\mathbf{A l}}$} & \multicolumn{1}{c|}{ Volume } & \multicolumn{1}{c|}{$\boldsymbol{\sigma}_{\mathrm{Al}}$} & \multicolumn{1}{c|}{$\boldsymbol{\sigma}_{\mathbf{M g}}$} \\
\hline 0.0 & 173.00 & - & 5.613 \\
\hline 0.09 & 167.25 & 5.303 & 5.617 \\
\hline 0.32 & 154.22 & 5.321 & 5.613 \\
\hline 0.425 & 148.84 & 5.328 & 5.610 \\
\hline 0.625 & 140.64 & 5.338 & 5.604 \\
\hline 0.75 & 136.20 & 5.343 & 5.601 \\
\hline 1.0 & 128.00 & 5.353 & - \\
\hline
\end{tabular}

* Pelzel et. al. (1940), volume at 1000K.

Table 3

\begin{tabular}{|c|c|l|l|l|}
\hline Alloy & Temp & $\boldsymbol{C}_{\text {Cd/Al }}$ & Computed & Expt. \\
\hline $\mathrm{NaCd}$ & $673 \mathrm{~K}$ & 0.1 & 0.243 & 0.244 \\
\hline & & 0.2 & 0.376 & 0.366 \\
\hline & & 0.3 & 0.400 & 0.359 \\
\hline & & 0.4 & 0.338 & 0.321 \\
\hline & & 0.5 & 0.136 & 0.239 \\
\hline & & 0.6 & 0.046 & 0.143 \\
\hline & & 0.7 & 0.270 & 0.053 \\
\hline & & 0.8 & 0.840 & -0.010 \\
\hline & & 0.9 & 0.670 & -0.023 \\
\hline $\mathrm{AlMg}$ & $1073 \mathrm{~K}$ & 0.09 & 0.274 & 0.270 \\
\hline & & 0.32 & 0.590 & 0.525 \\
\hline & & 0.425 & 0.519 & 0.575 \\
\hline & & 0.625 & 0.585 & 0.585 \\
\hline & & 0.75 & 0.789 & 0.530 \\
\hline
\end{tabular}

* Hultgreen et. al. 1973

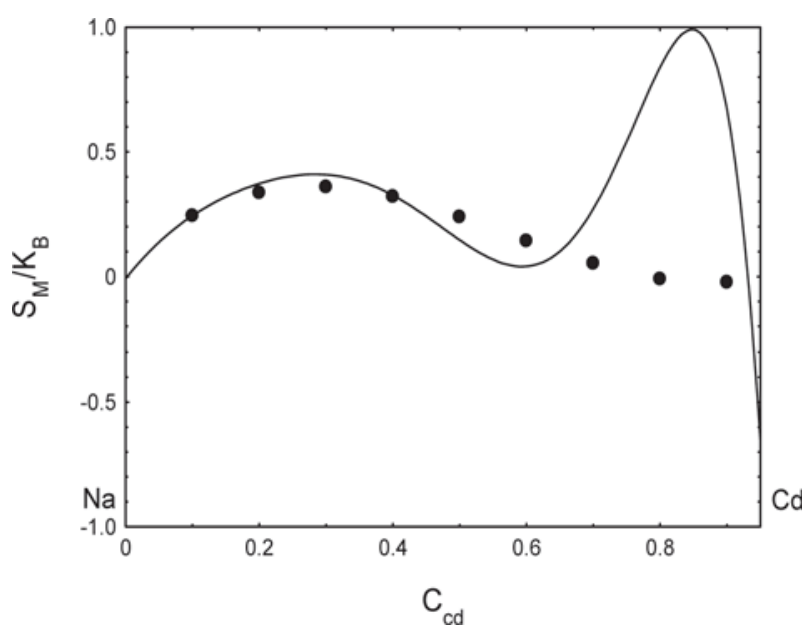

Fig.1: $\mathrm{S}_{\mathrm{M}} / \mathrm{K}_{\mathrm{B}} \mathrm{vs} \mathrm{C}_{\mathrm{cd}}$ for $\mathrm{NaCd}$ liquid alloys at $673 \mathrm{~K}$ (solid lines): theory; (closed circles): experiment (Hultgren et. al. 1973).

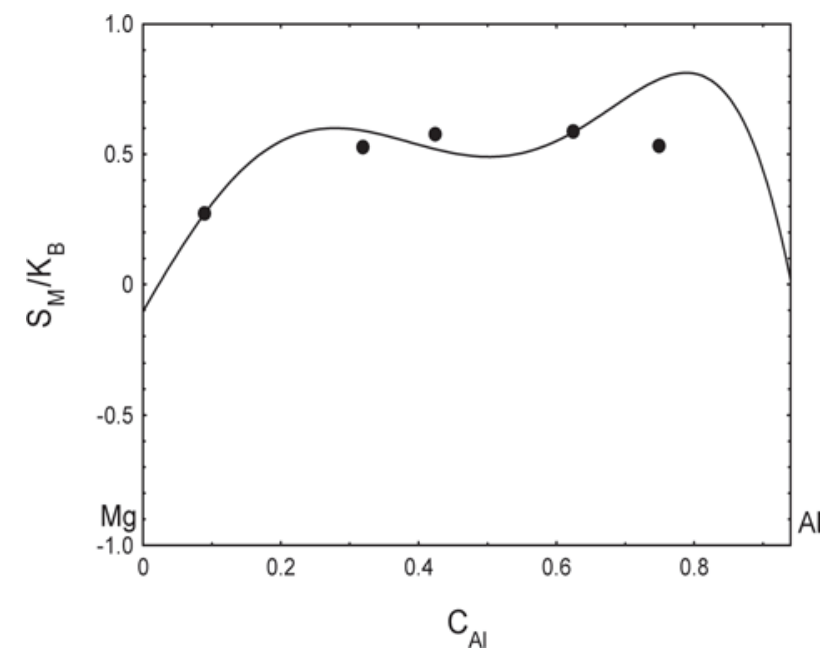

Fig.2: $\mathrm{S}_{\mathrm{M}} / \mathrm{K}_{\mathrm{B}}$ vs $C_{\mathrm{Al}}$ for AlMg liquid alloys at $1073 \mathrm{~K}$ (solid lines): theory; (closed circles): experiment (Hultgren et. al. 1973).

Due to the complexities involved in the determination, we have treated it as a parameter and fixed it as $\left(21.0 K_{\mathrm{B}}\right)$ for $\mathrm{NaCd}$ and $\left(27.8 K_{\mathrm{B}}\right)$ for AlMg alloys.

The computed and experimental (Hultgreen et. al. 1973) values of $S_{\mathrm{M}}$ for $\mathrm{NaCd}$ and $\mathrm{AlMg}$ systems are tabulated in Table 3 .

The $S_{\mathrm{M}}$ vs $C_{\mathrm{Cd}}$ curve for NaCd alloys, (Fig.1) shows good agreement between the computed and experimental values in the concentration range $0.1 \leq C_{\mathrm{cd}} \leq 0.6$, beyond this concentration range a distinct deviation in magnitude of $S_{\mathrm{M}}$ is observed. The theoretical values are all positive, where as the experimental values (Hultgreen et. al. 1973) are slightly negative in the concentration range $0.8 \leq C_{\mathrm{ed}} \leq 0.9$.

As for AlMg alloys, computed values agree well with the experimental as shown in (Fig 2). $S_{M}$ has been found positive at all concentrations.

The disagreement between computed and the experiment might be due to parameterisation of $\sigma_{3}$ and $\psi_{\text {comp. }}$. 
$S_{\text {comp }}$ which is used in equation (13), is the contribution from the internal degrees of freedom of the complexes, which is due to the internal modes of vibration. It is approximated by a sum of contribution from each complexes, $S_{\text {comp }}$ is used in equation (14), to get the entropy of mixing value, where $\psi_{\text {comp }}$ value is used as a parameter, which may not have explained the internal modes of vibration of the complexes exactly. So, in our work, we have concluded this as a major cause of deviation in case of $\mathrm{NaCd}$ alloys at higher concentration. Attempts are being made to improve our theory to give better results.

\section{CONCLUSION}

Our theoretical study of Na-Cd/Al-Mg alloys, which assumes the existence of $\mathrm{Cd}_{2} \mathrm{Na}$ and $\mathrm{Al}_{3} \mathrm{Mg}_{2}$ compound in the melt, explain successfully the concentration dependent asymmetry in the entropy of mixing.

The computed values of $S_{\mathrm{M}} / K_{\mathrm{B}}$ are positive at all concentrations, showing the atomic order. There are reasonable agreement between computed and experimental values in the concentration range of $0.1<C_{c d}<0.6$, beyond this range a distinct deviation in magnitude of $\mathrm{S}_{\mathrm{M}} / \mathrm{K}_{\mathrm{B}}$ is observed, for $\mathrm{NaCd}$ liquid alloy. For $\mathrm{AlMg}$ liquid alloys, computed values agree well with experimental values.

The deviation in $S_{\mathrm{M}} / K_{\mathrm{B}}$ values observed might be happening due to parameterization of $\sigma_{3}$ and $\psi_{\text {comp }}$, however attempts are being made to improve the pseudopotential theory so that it could be applied to strong interacting binary system.

\section{ACKNOWLEDGEMENT}

The authors are grateful to Prof. R. N. Singh, Department of Physics, Sultan Quaboos University, Muscat, OMAN for his valuable suggestions and discussions.

\section{REFERENCES}

1. Singh, R.N. 1987. Can. J. Phys. 65: 309.

2. Bhatia, A. B. and Hargrove, W. H. 1974. Phys. Rev. B. 10: 3186.

3. Prasad, L. C., Mikula, A. 2000. J. Alloys Compd.299:175.
4. Prasad, L. C., Chatterjee, S. K. and Singh, V. N. 1996. Physica. B 217: 285.

5. Sommer, F. J. 1990. Non-Cryst. Solids 117/118 : 505.

6. Bhatia, A. B. and Singh, R. N. 1982. Phys. Chem. Liq. 11: 285.

7. Young, W. H. 1992. Rep. Prog. Phys. 55:1769.

8. Lai, S. K., Matasura, M. and Wang, S. 1983. J. Phys. F. Met. Phys. 13: 2033.

9. Singh, R. N. 1981. J. Phys. F.Met. Phys. $11: 389$.

10. Haffner, J. 1977. Phys. Rev. A $16: 351$.

11. Hoshino, K. and Young, W. H. 1980. J. Phys. F. 10:1365.

12. Hoshino, K. and Young, W. H. 1981. J. Phys. F. 11: L7.

13. Hoshino, K. and Young, W. H. 1981. J. Phys. F. 11: L193.

14. Nguyen, V.T. and Enderby, J. E. 1977.Phil. Mag. 35:1013.

15. Vander Marel, C., Van Oosten, A. B., Geerstma, W. and Van der Lugi, W. 1982. J. Phys. F. 12: 2349.

16. Saboungi, M. L., Marr, J. and Blander, M. 1978. J. Chem. Phys. 68: 1375 .

17. Hultgreen, R., Desai, P. D., Hawkins, D. T., Gleiser, M., Kelly, K. K. and Wagman, D. D. (American society of Metals, Ohio 1973). Selected values of the thermodynamic properties of the Binary alloys.

18. Mcalister, S. P. and Crozier, E. D. 1974. J. Phys. C. 7:3509.

19. Jha, I. S., Singh, R. N., Shrivastava, P. L. and Mitra, N. R. 1989. Phil. Mag. C $1: 15$.

20. Prasad, L. C., Chatterjee, S. K. and Jha, R. K. 2007. J. Alloys and compounds. 441: 43.

21. Prasad, L. C., Singh, R. N., Singh, V. N. and Chatterjee, S.K. 1995. Physica. B 215:225.

22. Chatterjee, S. K. and Prasad, L. C. 2004. Indian Journal of Pure \& Applied Physics. 42: 279.

23. Wang, S. and Lai, S. K. 1980. J. Phys. F. Met. Phys. 10: 2717.

24. Herzberg, G. 1962. Molecular, spectra and Molecular Structure, 2.

25. Chatterjee, S. K., Prasad, L. C., and Bhattarai, A. B. 2010. J. Alloys and compounds. 496: 100. 